\title{
Herlyn- Werner- Wunderlich Syndrome: A Rare Urogenital Anomaly Masquerading as Acute Abdomen
}

Sir,

A 14-yr-old girl presented with chief complaint of lower abdominal pain during menses for last 1 year. Her menstrual history suggested no abnormality except dysmenorrhea. On examination secondary sexual characters were normal for age. Per abdomen examination and rest of the systemic examination was also normal. A provisional diagnosis of primary dysmenorrhea was made and the patient counseled accordingly. She was advised to take anti-spasmodic drugs during her menstrual periods and follow up monthly.

One month later she presented in emergency department with acute retention of urine and lower abdominal pain. After appropriate clinical examination, an urgent ultrasonography was done which revealed a bicornuate uterus with hematocolpos (80 c.c of fluid) and non visualization of right kidney, with left kidney being normal. MRI was done to assess anatomic conformation. It was suggestive of uterus didelphys with double cervix and vagina with hydro/ hematocolpos in right sided vagina. Vagina of left side was separated from the right one by a blind vertical septum. (Fig. 1).

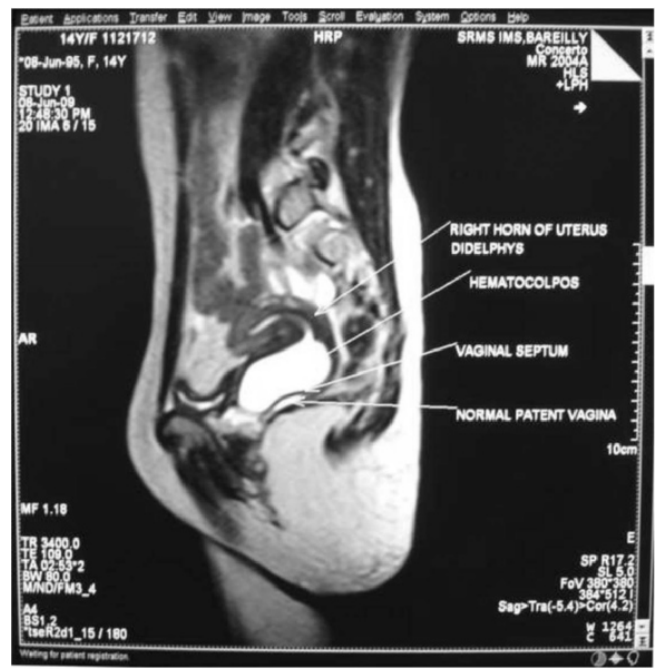

Fig. 1. MRI shows Right horn of uterus didelphys communicating with hematocolpos. The vagina on left side is separated by hypo intense septa from right side and is collapsed.

The patient was taken up for examination under anesthesia. The septum was excised transvaginally and hematocolpos drained. Both the cervices could be visualized after resection of the septum. The patient became symptom free after the procedure.

Congenital anomalies of the Mullerian duct system can result in various urogenital anomalies and HerlynWerner- Wunderlich syndrome is one such rare anomaly characterized by uterus didelphys with blind hemivagina and ipsilateral renal agenesis. ${ }^{1}$ The diagnosis in this condition is usually made after menarche but its rarity and varied clinical presentation may contribute to diagnostic delay, for years after menarche. The initial presenting signs and symptoms were followed by an incorrect diagnosis by the referring physicians $100 \%$ of the time, as also missed by us. ${ }^{2}$ The most common clinical presentation is that of pelvic pain shortly after menarche, in association with vaginal or pelvic mass, with normal menstrual periods, as also reported by Jindal et al. ${ }^{3}$ Rarely, it may present as abnormal discharge, infertility and acute abdomen as well.

For diagnosis, ultra sonography usually gives a correct picture, by showing uterovaginal duplication, hematocolpos or hematometrocolpos along with the absence of ipsilateral kidney. ${ }^{4}$ However MRI has been considered as the imaging modality of choice by various authors. ${ }^{5}$

Transvaginal excision of the septum, large enough to allow a permanent drainage of the menstrual blood from the hemiuterus, is the appropriate mode of treatment as soon as the condition is diagnosed. Early and accurate diagnosis of this condition is necessary by the physicians, so that prompt and adequate surgery can be done to provide relief from the pain and prevent further complications of infertility, endometriosis and pyocolpos.

Ashu Rastogi and Anshu Khamesra ${ }^{1}$ Departments of Pediatrics and ${ }^{1}$ Obstetrics and Gynaecology, SRMS Institute of Medical Sciences, Bareilly 243001 (U.P),

India.

E-mail:drpaed@rediffmail.com [DOI-10.1007/s12098-010-0148-4]

\section{REFERENCES}

1. Sarac A, Demir MK. Herlyn- Werner- Wunderlich syndrome: a rare cause of infertility. Eur Radiol 2009; 19: 1306-1308.

2. Zurawin RK, Dietrich JE, Heard MJ, Edwards CL. Didelphic uterus and obstructed hemivagina with renal agenesis: case report and review of literature. J Pediatr Adolesc Gynecol 2004; 17: 137-141.

3. Jindal G, Kachhawa S, Meena GL, Dhakar G. Uterus didelphys with unilateral obstructed hemivagina with hematometrocolpos and hematosalpinx with ipsilateral renal agenesis. J Hum Reprod Sci 2009; 2: 87-89.

4. Orazi C, Lucchetti MC, Schingo PM, Marchetti P, Ferro F. Herlyn-werner-wunderlich syndrome: uterus didelphys, blind hemivagina and ipsilateral renal agenesis. Sonographic and MR findings in 11 cases. Pediatr Radiol 2007; 37: 657-665.

5. Mirkovic L, Ljubic A, Mirkovic D. Magnetic resonance imaging in the evaluation of uterus didelphys with obstructed hemivagina and renal agenesis: a case report. Arch Gynecol Obstet 2006; 274: 246-247. 